\title{
Newcomer challenges with accessing healthcare services in Saskatchewan, Canada
}

\author{
Ginny Lane ${ }^{1}$, Mayari Hengstermann ${ }^{2}$, Judy White ${ }^{3}$ and Hassan Vatanparast ${ }^{4}$
}

\begin{abstract}
Newcomers experience a variety of barriers to healthcare services. The study objective is to identify newcomers' healthcare barriers. During 2014-2015, we conducted in-depth interviews with service providers, and newcomer parents who had been in Saskatchewan for under 5 years. Primary themes include: 1) navigation difficulty, 2) limited awareness, 3) language, 4) interpretation and translation, 5) bealth benefits, 6) service limitations and stigma, 7) gender and cultural concerns, 8) bealth attitudes and beliefs, and 9) work demands. The results exposed service gaps and suggest options to improve access to healthcare. Consideration should be given to the development of formal processes to ensure the provision of information concerning healthcare and health benefit programs, as well as responsive healthcare services, including convenient primary healthcare sites that offer comprehensive care in a culturally responsive manner with embedded interpretation services to ensure that the Saskatchewan healthcare system does not perpetuate or create health disparities.
\end{abstract}

Keywords: Immigrant; refugee; healthcare access; cultural competency; healthcare barriers

\section{Introduction}

Access to healthcare is a central component in the promotion of health and wellbeing among all Canadians, including newcomers. Both immigrants and refugees may experience a variety of barriers to accessing healthcare services including low health literacy (Renfrew et al., 2013), lack of familiarity with the healthcare system (Neufeld et al., 2002; Wu et al., 2005; Son, 2013), cultural competency of healthcare professionals (Hansson et al., 2009), cost (Access Alliance, 2011), traditional beliefs (Renfrew et al., 2013, Council of Agencies Serving South Asians, 2008), language preference (Renfrew et al., 2013, DuBard \& Gizlice, 2008), limited appointment times (Renfrew et al., 2013), and lack of childcare (O’Mahony \& Donnelly, 2007; Sethi, 2013) or transportation (Kilbride, 2010). Refugees may also experience a sense of discrimination and stigmatization that deters them from accessing healthcare (Szajna \& Ward, 2015). In addition to the above barriers, recent Syrian refugee parents have reported that preoccupation with previous trauma, depression and anxiety, and social isolation were barriers to seeking healthcare for their children (Alwan, Schumacher, Cicek-Okay, et al., 2020).

Cost and lack of provincial healthcare insurance coverage can be strong deterrents to seeking healthcare as newcomers must endure a 3-month waiting period before they are eligible for

\footnotetext{
${ }^{1}$ Ginny Lane, University of Saskatchewan, Saskatoon, Saskatchewan, Canada. E-mail: virginia.lane@usask.ca.

${ }^{2}$ Mayari Hengstermann, Universidad del Valle de Guatemala, Guatemala City, Guatemala.

E-mail: mayarihengstermann@gmx.de.

${ }_{3}^{3}$ Judy White, University of Regina, Regina, Saskatchewan, Canada. E-mail: Judy.White@uregina.ca.

${ }^{4}$ Hassan Vatanparast, University of Saskatchewan, University of Saskatchewan, Saskatoon, Saskatchewan, Canada.

E-mail: vatan.h@usask.ca.
} 
provincial healthcare insurance in some provinces, while undocumented immigrants are not eligible for provincial healthcare insurance in any province (Access Alliance, 2011). Newcomers have described the challenge of paying for hospital care when they lack provincial healthcare insurance and the burden of paying for user fees and prescription drugs on meagre incomes (Access Alliance, 2011).

Culture and language limitations can impact access to healthcare. Newcomers often prefer to access healthcare from service providers who are from the same culture and speak their language and will travel farther to see them (Son, 2013), but this ideal is not always possible (Munger et al., 2010). Language barriers can result in misunderstandings that lead to misdiagnosis, poor treatment plans, and unnecessary referrals (Goggins, (2008). These poor experiences can have detrimental impacts on health status, create inefficiencies in the healthcare system, and result in service avoidance.

Some newcomers may be reluctant to access certain types of healthcare services due to cultural beliefs about health, illness, and treatments. They may prefer to access traditional healthcare approaches, instead of biomedical healthcare (Council of Agencies Serving South Asians, 2008). Responses to illness can be deeply influenced by social perceptions of health and illness (Hengstermann, 2016). They may forgo accessing preventive care because they do not recognize its role to maintain good health (Vargas Bustamante etal., 2010) or manage chronic conditions (Renfrew et al., 2013). Newcomer women are at risk for not accessing healthcare due to a number of intersecting factors such as cultural or religious practices that forbid seeking services from providers of the opposite sex (Weerasinghe \& Mitchell, 2007). This leads to reluctance to attend health appointments due to culturally inappropriate services (Sethi, 2013). These diverse cultural beliefs and reticence to access preventive care may result in delayed access until the disease is more advanced.

Once a decision is made to access healthcare, newcomers may need to overcome several practical barriers to attend an appointment. They may have difficulty with taking time off work or arranging transportation (Council of Agencies Serving South Asians, 2008; Sanmartin \& Ross, 2006). Newcomer women and temporary residents on work visas may be especially reticent to take time off work (O’Mahony \& Donnelly, 2007; Yun et al., 2013). Newcomers may require additional support to identify healthcare services with extended hours or more convenient locations.

Although much is known about the barriers to healthcare that newcomers experience, most research is based in large cities with significant newcomer populations and healthcare services that have developed some expertise to serve them. This is likely the first study of this type done in Saskatchewan, Canada, which has only recently experienced a surge in immigration and has not developed the array of services found in Toronto or Vancouver. This article explores newcomer families' challenges with accessing healthcare during their first five years in Saskatchewan, Canada to inform future healthcare investments.

\section{Methods}

This study is part of the larger multi-method Healthy Immigrant Children study using both quantitative and qualitative methods to yield complementary data to support a more complete description of the health status of newcomer children (Lane et al., 2018). 


\section{Methodological Approach}

Focused ethnography was employed to analyze newcomer access to healthcare in Saskatchewan, Canada. According to Knoblauch (2005), a focused ethnography involves studying a distinct problem in a specific context among a sub-cultural group that may not be completely foreign to the researcher. In accordance with focused ethnography, the research focused on common behaviors and shared experiences among newcomers to capture cultural perspectives and to make practical use of that understanding in relation to healthcare access (Cruz \& Higginbottom, 2013). In addition, the study was characterized by a specific research question, led by a researcher with insider knowledge of the healthcare system and cultural capacity issues, and involved intensive in-depth interviews that took one to two hours (Higginbottom, 2013). Focused ethnography provides a framework to understand cultural patterns of behavior related to healthcare access and their underlying customs, ideas, beliefs, and knowledge; as well as to expose power, control and inequality issues (Roper \& Shapira, 2000; Smith, 2001). Focused ethnography has been described as ideal for studying emerging cultural contexts (Knoblauch, 2005), such as the relatively new phenomena of newcomers accessing the Saskatchewan healthcare system.

The lead researcher's starting point is as a Canadian-born researcher of Western European descent with significant experience living and working in Latin America. She has over 20 years of experience working in healthcare at both the front line and policy levels, which provides insider knowledge, leading to an awareness that the population is rapidly changing, but services are slow to respond to changing needs. During the study, she bracketed expectations and engaged as a sociologist with a healthcare background.

\section{Participants}

Several newcomer settlement organizations, such as Open Door, assisted with participant recruitment by inviting newcomer parents to participate. Participants had been in Canada for less than 5 years and were living in either Regina or Saskatoon. A purposefully selected sample of 19 refugee and immigrant parents who reflected the typical ethnic origins of recent Saskatchewan newcomers participated. In addition, a purposefully selected sample of 24 newcomer service providers (settlement agencies, community schools, English-as-a-secondlanguage programs), healthcare providers, and policymakers participated. Participants provided written consent and the University of Saskatchewan Research Ethics Committee provided ethical approval.

\section{Data Collection and Analysis}

The in-depth interviews about healthcare access focused on awareness of the Canadian healthcare system, and barriers and supports that may affect access to healthcare. The interview questions are available in appendix A. The interview questions were modified from existing questionnaires whenever possible (Weerasinghe \& Mitchell, 2007; Groleau \& Kirmayer, 2004; Young et al., 1999). Interviews used open-ended questions to facilitate the collection of rich, descriptive narratives (Morse \& Field, 1995) until further interviews did not yield additional significant information, indicating saturation had been reached. Interviews were conducted in English by the same researcher. Interpreters were available, but were only required on one occasion, as most participants did not require one. Some newcomer 
organizations designated 2 or 3 staff to participate, so they were interviewed as a small group using the same questions and transcribing answers with the speaker noted.

Interviews were approximately one-hour long. They were recorded and transcribed verbatim following the sessions and rechecked at minimum a second time by the same person. Transcripts were analyzed to identify recurring words, phrases, and ideas corresponding to the research question, which were then grouped into themes (Mayan, 2009). An inductive approach with open coding of early data was used to generate categories embedded in the data (Green \& Thorogood, 2007). Themes were identified by referring to both the number of similarly coded data extracts, as well as divergent experiences of some importance. Deviant cases were thoroughly reviewed to capture diverse participant experiences. Early interviews were conducted concurrently with data analysis in an iterative process to allow for initial results to be used to open up additional lines of investigation and further fine-tune questions and participant probing.

A second person reviewed the transcripts to determine whether there was agreement with coding decisions. Any discrepancies were discussed among the authors, and mutual agreement reached. Themes were further refined by combining categories and organizing sub-themes under them. NVivo11 was used to facilitate qualitative data analysis.

Rigor (trustworthiness) was achieved through attention to validity (credibility and transferability) and reliability (dependability and confirmability) (Cypress, 2017; Morse, 2015). With regards to validity, thick description was achieved through prolonged engagement, as participants had attended 3 previous appointments with the research team to gather data for other parts of the study, probing to expand on answers, and attaining data saturation; as well as by including data from service providers, which theoretically increased the sample size as they were drawing on their experience with a large number of newcomers. The purposeful sampling used to draw a sample that represents the ethnic origins of recent newcomers to Saskatchewan ensured transferability of results in the Saskatchewan context. Including participants from the two groups also increased validity by allowing for triangulation of data from 2 different sources (Cypress, 2017).

Reliability was assured through using interpretive coding by one researcher. Coding decisions were made in the context of the knowledge gained from all previous interviews, as described by Morse (Morse, 2015), which were then confirmed by a second researcher. Researcher bias was minimized by bracketing researcher beliefs, asking about both positive and negative experiences with healthcare, using an inductive analytic approach, and taking time to carefully reflect following each interview (Cypress, 2017). Internal reliability was demonstrated by overlapping interview data from different participants (Morse, 2015).

\section{Results and Discussion}

All newcomer participants were parents of children between the ages of 3 and 13 years. Fifteen mothers and 7 fathers from 19 distinct family units participated. In 3 cases both the mother and father participated as a couple. Participants included 13 immigrants and 9 refugees. They were from the United States (2), Western Europe (2), Eastern Europe (1), Latin America (1), Africa (2), Asia (6), and the Middle East (8). Families from the US and Western Europe reported high incomes, one Middle Eastern and one Latin American family reported middle incomes, while all others reported low incomes. Service providers were not asked to provide 
demographic information. Nine primary themes related to healthcare access emerged. Each theme draws on newcomer parent and service provider data when available.

\section{Theme 1: Navigation difficulty}

Overall, the greatest barrier to accessing healthcare services was health system navigation difficulties. Participants talked about difficulties with understanding how and where to access care, as well as problems with how the health system interacts with patients, which created confusion and delays. The son of a refugee shared:

We called our doctor's office and she said we can't see you right now, it will be 8 days... My arm got broken at the night and I can't wait 8 days, but in the morning we just went to the family doctor's office and they sent me to the walk-in clinic, then to $\mathrm{x}$-ray, walk-in clinic and they gave me a sling. (SR1)

Some newcomers experienced difficulties with following through with referrals due to not fully understanding the process or recognizing the importance of the appointment, resulting in fragmented care. Healthcare providers did not always fully understand or take the time to check with the newcomer's family physician to ensure an accurate medical history, which can have tragic results as described by a healthcare provider:

Glucose 6-Phosphate Dehydrogenase Deficiency (G6PD)...if these people are exposed to certain medications or food...they could develop severe hemolysis and kidney failure and a lot of people from the Burmese group and Bhutanese group have G6PD...we made alert cards for this people... any time you get medication from a pharmacy, you see a different physician... you have to show it to them...I gave the card to parents...their child was very sick and end up in the hospital and they gave him something, maybe prophylactic treatment for TB... and the kid almost died... (RHP1)

Some newcomers had trouble with the process of finding a family physician. An immigrant from the Middle East stated:

On health region website there is one doctor... and two times I tried to call her, her office talked with me and she was not there, so I didn't try again. I thought I need to talk with the doctor (SI1).

Notably, various newcomers, not only those of minority ethnic origin, expressed difficulties with finding a family physician.

Current study results align with Woodgate et al. (Woodgate et al., 2017), a study based in Manitoba, Canada, that demonstrated recent newcomers do not always understand how or where to access healthcare services. In the United States, Syrian refugee parents also reported numerous difficulties with navigating the healthcare system, often resulting in missed appointments for their children (Alwan et al., 2020)

\section{Theme 2: Limited awareness of healthcare services}

A few service providers and newcomers specifically mentioned not being aware of healthcare services. Many newcomers did not expect some healthcare services to be available simply 
because they are not offered in their home country, such as home care services. An immigrant service provider shared:

Some (newcomer) children have cerebral palsy...they are getting bigger...the mothers ...take care of them at home... does not get home care, doesn't even know about home care... Unless the doctors tell them they don't know forever. Newcomers don't even know to ask (RIS1).

Newcomers spoke about relying on family members, friends, neighbours or their settlement counselors for advice on where to seek healthcare when the need arose. An immigrant from the Middle East mentioned:

...we don't have any...family members or...friends when we come to Regina, we had no contacts, it takes time to know your community centres" (RI1). They often reported being unprepared for knowing where to seek services when illness struck the family.

\section{A refugee from Asia stated}

... when we just arrived I didn't know how to go anywhere and when my family is sick I had to run to Open Door to talk to the caseworker (RR1).

Several studies indicate lack of familiarity with the healthcare system is a barrier to accessing healthcare among newcomers (Wu et al., 2005; Son, 2013). A Syrian refugee parent noted that if a child is sick, they did not understand where to take the child to access appropriate care (Alwan et al., 2020). Similarly, Syrian refugees in Toronto, Canada expressed concerns regarding lack of accurate and user-friendly information about healthcare services (Guruge, Sidani, Illesinghe et al., 2018). These issues underline the importance of consistent healthcare orientation designed for individuals with limited English skills and low literacy levels (Renfrew et al., 2013).

\section{Theme 3: Language difficulties, low literacy}

Language difficulties were mentioned by service providers with the same frequency as navigation difficulties, and were the third most common barrier named by newcomers (tied with healthcare service limitations, stigma). Service providers described their difficulties with both making appointments with newcomers and having appropriate communication that resulted in shared understanding. An immigrant service provider commented:

They have to go with interpreter to learn about diet, if they don't they don't understand ...they keep eating what they have been eating...If there is a very complicated health issue... high blood pressure, cholesterol and blood sugar, many need help to go see doctor very often... frustrating for families...not understanding anything. (RI1).

Despite the impact that language barriers can have on healthcare, many newcomers stressed how they coped by relying on the assistance of family and friends who spoke English, as described by an immigrant from the Middle East, "When we were new language was an issue. My brother-in-law helped interpret for us. Then I started language class. I had to depend on my brother-in-law at the beginning" (SI2). Newcomers thus accepted some responsibility for 
fitting into the English speaking healthcare system. However, some newcomers found physicians who spoke their native language.

Many service providers noted that some newcomers have lower literacy levels. According to a healthcare provider, “...82\% (of clients) have a health literacy rate of 2 or below (at an inner city clinic, which indicates) capacity to deal with only simple, clear material involving uncomplicated tasks" (SHP2). Diabetic newcomers with lower literacy skills did not fully understand how to match carbohydrate consumption with medication dose to maintain a healthy weight. A refugee from Asia shared, "She (diabetic wife) eats normally, just avoids sweets. The ...nutritionist talked to her about food also, but we forget, only remember eat healthy food, vegetables, milk, sandwich ... She loses weight all the time" (RR1).

Health literacy refers to an individual's capacity to understand, communicate, and process basic health information to inform healthcare decisions. Renfrew et al. (2013) have observed that low health literacy is a significant barrier to care among newcomers. Syrian refugees have experienced difficulty with comprehending diagnoses, treatment plans, and medication administration (Alwan et al., 2020).

\section{Theme 4: Interpretation and translation issues}

Closely linked to language difficulties are interpretation issues, as suggested by both service providers (third most common barrier) and newcomers (fifth most common barrier). Many participants talked about difficulties with accessing appropriate interpretation services. Although refugees had to access some funded interpretation services, immigrants did not. Several healthcare service providers stressed how access to telephone translation has been very helpful; however, it was not consistently used, nor available in all facilities. Although phone interpretation services can be useful, there are limitations, as recounted by a refugee from Asia: "I took my child to university hospital... an interpreter available by phone and the phone got disconnected and it would be easier if the person was available right in front of us" (SR2).

Sometimes children were relied on as healthcare interpreters, which is not necessarily appropriate, as mentioned by a service provider:

... a grade 8 student, her mom had to go to the doctor and she recently had a baby and has some personal medical things...she takes her daughter with her... She pulls her out of...school to go to these appointments to help and I don't know if it is always in the best interests of the child for them to be in medical appointments and hear it all (SCP1).

In some cases, the interpreter was able to literally interpret, but did not share the same cultural understanding as the client and misunderstandings arose. Lack of access to an interpreter or poor interpretation led to delayed healthcare access, inappropriate treatments, poor health outcomes and ethical dilemmas. The availability of quality interpretation services helped to ensure accurate assessment as emphasized by an immigrant service provider:

...I was interpreting for the patient and he (doctor) said this is the first time I saw her talking... before only her husband was telling me... when the doctor saw how she was interacting with me he said it made a big difference on how to prescribe the medication... Her husband was not actually translating, just speaking on behalf of 
her...When she is talking in Bengali language to the interpreter the doctor can read all the expression of the body language and (it) helped his assessment (SIS2).

In the United States, Syrian refugees have described similar challenges with translation services, including differences in colloquial Arabic dialects among interpreters (Alwan et al., 2020). Interpretation issues noted underscore the growing importance of using medically trained interpreters. Juckett and Unger (2014) have reported the use of professional interpreters is superior to the common practice of using family, friends, or untrained staff as interpreters to reduce risk for errors, violation of confidentiality, and poor outcomes. In addition, they recommend that children should never be used as interpreters, except in case of an emergency.

\section{Theme 5: Difficult access to health benefits}

Although all newcomers with permanent resident status were immediately eligible for a Saskatchewan health card upon arrival, refugee claimants or individuals who were here on a temporary worker visa experienced some gaps in coverage. An immigrant service provider recalled, "...a temporary worker...his permit was expired and he was waiting for the application process... his health card was expired...He could only go to Emergency...they can't deny him access, but they can bill him if they provide service" (RIS2).

When government-assisted (GA) refugees arrived, they were eligible for Interim Federal Health Benefits (IFHB); however, due to policy changes and court challenges around eligibility, some healthcare providers were reluctant to provide services during the study period. An immigrant service provider observed, "There are some dentists and pharmacies who are reluctant to do IFHB work because of the paperwork and uncertainty of getting paid" (SIS1).

Newcomers frequently spoke about their concerns with accessing health services that were covered by provincial health benefits programs for individuals with lower incomes (second most common barrier) such as Family Health Benefits and Special Support Program for Drug Coverage. While some refugees were well supported by caseworkers to access health benefits, not all refugees experienced a smooth transition to Family Health Benefits when they started working, and experienced financial pressures from high drug costs or serious difficulties with accessing dental care for their children. A refugee from Asia shared:

... when I was on social assistance no big charges sometimes when I buy drugs, $\$ 1$, $\$ 2$ or $\$ 3$, it is good. But I start to work... and after that whatever I have to buy I pay full charge. I didn't know about Family Health Benefits...now I have to pay for the amoxicillin for $\$ 20$ or $\$ 40 \ldots$ no more supplementary health benefits (SR2).

In addition, sometimes provincial health coverage programs were insufficient for individuals who required high-cost drugs. According to an immigrant service provider:

.... client mother and son with HIV ... medication is very expensive and she has to pay $2 \%$...she was paying $\$ 149$ every month...from her food allowance and... the child tax benefit...very expensive medication...I applied for her under Saskatchewan Drug program, still they said only she will pay the $2 \%$, this is mandated...is a lot for her (SIS2). 
When immigrants arrived, they were expected to be self-supporting and often lacked the guidance of a settlement counselor to advise them on how to access health benefit programs. Many immigrants mentioned difficulties with accessing dental and optometric services due to lack of knowledge about health benefits. An immigrant from the Middle East recounted:

Family Health Benefits, we didn't know that from any organization, we come to know by one of our family friends... After a long time...6 months, more...We applied for special support for drug coverage and then they determined we were also eligible for Family Health Benefits...no job or anything so the first few 6 months are difficult...(RI1).

In contrast to the experiences of many newcomers, a white male immigrant from the United States did not hesitate to approach a dentist when he needed care, and he was treated fairly with consideration given to his lack of dental insurance. He stated:

...I had broken a tooth...I was a consultant so didn't have supplemental insurance... The dentist figured out what the insurance was and then asked me how much I could pay and wrote off all the rest and I had just met the man...He could have probably charged twice what he did (RI2).

Similar to the experiences shared above, Woodgate et al. (2017) mentioned that the cost of medications and non-essential care was very high for low income newcomer families that could barely afford their monthly bills in Manitoba, Canada. Similarly, Syrian women refugees living in Toronto, Canada that were nearing the end of their one-year sponsorship period, which included supplemental and prescription drug coverage, expressed concern about future healthcare costs, including medications, physiotherapy, and dentists (Guruge et al., 2018).

\section{Theme 6: Healthcare service limitations, stigma}

Service providers and newcomers both recognized limitations with healthcare services, such as appointment-based care or short appointment times, which can result in suboptimal care and reduced client satisfaction. Many newcomers mentioned difficulties with trying to make time in their busy schedules to attend healthcare appointments, as well as with fully addressing their health issues at an appointment. A refugee from Asia explained, "I go to the...walk in clinic when they are sick because it is closer to our area. Usually getting an appointment over at the... (primary care) clinic is not easy" (RR3). A refugee from Asia shared, "I have day off on Friday, but...there is not any TB check clinic on Friday for refugees" (SR3).

Some healthcare providers also recognized that they did not have enough time to spend building relationships with clients. A healthcare provider explained, “...take time...you can't expect them to go to the clinic and run through things like, snap, snap... You have to put the human individual first and the relationship develops... once you have that relationship going the people are open and you can work better" (SHP3).

Service providers mentioned that accessing some healthcare services, such as mental health, carries a stigma for newcomers that may prevent optimal access to appropriate care as recounted by a healthcare provider, “...sending these people to mental health was a big challenge and with the stigma they wouldn't show up for their appointments" (RHP1). An added dimension was that newcomers were reluctant to attend specific clinics if they were viewed as being designated for First Nations people. An immigrant service provider 
mentioned, "Newcomers don't like going to (clinic) because they hear negative things about First Nations people attending that clinic. They adopt these stereotypes" (SIS1)

Results indicate the design of our healthcare system to efficiently address healthcare concerns through an appointment-based system does not meet the needs of newcomers who may need additional time, and convenient or flexible appointment times. This is similar to Renfrew et al. (2013), who observed that limited appointment times are a barrier to care. Previous research has also identified significant stigma associated with accessing mental health services (Karago-Odongo, 2008). In addition, current participant comments indicated that creating a First Nations client-centered environment may be alienating them from accessing those services.

\section{Theme 7: Gender and Cultural Concerns}

Although many service providers perceived that gender and cultural concerns were a common barrier to healthcare (fourth most common barrier), it was less commonly mentioned by newcomers (sixth most common barrier). Healthcare providers perceived that the healthcare system lacks the capacity to respond to newcomer healthcare needs. A healthcare provider explained, "...the capacity of our healthcare providers in recognizing and understanding the needs of newcomers and being able to adjust their practice...requires additional time and resources and not everybody is comfortable with that process or willing to invest" (SHP3).

Cultural beliefs and attitudes of both healthcare providers and their newcomer clients influenced the process and outcomes of healthcare delivery. Misunderstandings occurred when individuals did not describe or exhibit symptoms that fit with Canadian expectations. An immigrant service provider described the case of:

...a woman... with a severe pain in her abdomen area and she was Muslim and it was fasting month...she went to the hospital only one doctor assessed her... and said the pain you are feeling is because you have been fasting and she was sent home...back to the hospital with severe pain... her appendix had burst (SIS3).

In some cases, healthcare providers did not realize they expressed biased cultural opinions and immigrant service providers acted as cultural buffers to assist clients with verbalizing their healthcare concerns. An immigrant service provider recounted, “...the doctor told her not to eat rice, just to eat brown bread and she said (to me) even...if she ate 10 bags of brown bread she would still feel hungry for rice. If rice is one big plate, it would make her full, but not brown bread" (RIS1). Service providers mentioned cases where newcomers' health concerns were not taken seriously by healthcare providers or they were not treated with dignity due to perceived bias or lack of trust, especially in cases involving immigrant physicians.

Some newcomers described ongoing health concerns that never seemed to result in a diagnosis or effective treatment, which may have been related to high stress due to integration problems, but healthcare providers did not recognize the possibility of a mental health issue. An immigrant from the Middle East commented:

...he (husband) took some medicine for his headache that our family doctor prescribed ... but he got reaction from that medicine and he got a stomach flu...2 months it was continuous...his back was sore, his whole body was sore, then headache, he was getting tired... again to the... walk in clinic and they gave him some 
medicine. It helped a bit, but not that much...now he sometimes gets chest pain also so doctor advised him that if he gets really bad then he can go to emergency. He doesn't know what's wrong (SI1).

Many service providers and newcomer women mentioned the importance of gender-based care and the associated challenges, such as incomplete examinations, resulting in poor assessment and outcomes. They spoke of challenges with finding a female physician. A Saskatoon immigrant commented, "I want a female doctor for me because I am not comfortable to see a male doctor... I have not found a female doctor yet" (SI1).

A Saskatoon immigrant service provider recounted the case of:

.... client...from the Middle East...I ask her why you are white...She told me, I am bleeding and it doesn't stop... for 4 months... he (physician) told her when the period comes it will stop. I sent her to a very respectful doctor, an immigrant doctor...I told him she is covered so you can't see her, so please prepare a gynaecologist female...he talked to the female Indian gynaecologist...they did a checkup...sent her to the hospital and they did a cleanup... She didn't have any blood in her body (SIS2).

In contrast, an American immigrant woman was not at all worried about seeing a male physician.

Some women lived in vulnerable circumstances that impacted their capacity to access care or to follow health care recommendations. Lack of awareness of these types of issues or the inability to respond appropriately created serious barriers to the provision of optimal care. An immigrant service provider mentioned the case of:

...(a) woman... from Africa, from a country that... if she couldn't have a boy, the husband had the right to go and remarry again and be pregnant. The woman had in a matter of 1 year...4 pregnancies that failed...she was so worried for her life she kept getting pregnant...we eventually sent her to a social worker (SIS3).

There were some problematic conversations about contraception and abortion between healthcare providers and newcomer women, likely due to healthcare provider bias and poor understanding of culturally-based views on the topic. An immigrant service provider shared:

.... a women here with an unwanted pregnancy and she happened to see a nurse in the hospital who was against abortion and with her broken English she figured that she should not do it...the way that they treated the woman she figured from her perspective that it was wrong (SIS3).

Participants' comments are consistent with previous research that noted newcomers often prefer to access healthcare from service providers of the same culture (Son, 2013), but find that this is difficult to achieve (Munger et al., 2010). Research has also observed that some newcomer women are bound by specific cultural or religious practices that require them to seek healthcare from female providers, which can be difficult (Sethi, 2013). Syrian refugee women in Toronto, Canada noted concerns about male interpreters being assigned to accompany them to reproductive health related appointments, which created a gender barrier to discussing their concerns with the doctor (Guruge et al., 2018). An additional dimension to some newcomer women's experiences is their insecure immigration status as the spouse of 
a temporary worker or economic dependence on their spouse, which can create challenges with accessing services (O’Mahony \& Donnelly, 2013).

\section{Theme 8: Health Attitudes and Beliefs}

Some newcomers came from environments in which they had limited access to preventative or long-term treatment of chronic conditions, so they only went to see the physician when they were so sick they could not work. An immigrant service provider stated:

Due to the limited access to the healthcare system in the refugee camp they never practiced going to their physician... or routine immunizations for their children... and they have the same kind of concept after coming to Canada; they will not go and see the doctor until they are in the final stage (RHP1).

Sometimes newcomers expected biomedical medicine to offer cures for all diseases and did not understand the concept of viruses that do not respond to antibiotics, or chronic disease that needs long-term treatment. A healthcare provider described how, “... most cultures think that the doctor gives me this medicine, when it is done I will feel better... don't know there is something called chronic disease and they have to take medicine for a long time" (RHP2).

Some newcomers relied on traditional health practices that may adversely interact with biomedical treatments. A healthcare provider described the case of a:

...(cancer) patient who was...bleeding...finally when it got to the point of being life threatening...realized that this individual was talking with a family member from their home country...recommending some supplements... on top of their medical treatment, but the supplements were actually acting like a blood thinner (SHP1).

Some service providers acknowledged that fear of government authorities or lack of trust in the public healthcare system could make it difficult to establish good relationships. Cases of domestic violence can be especially complicated to establish trust, as reported by an immigrant service provider, “....some don't like counseling for domestic violence...fear of authority, of being reported, of getting the husband in trouble, which will lead to him being sent home and the whole family will have to return with him" (SIS2).

In some cases, lack of trust in the public healthcare system can lead to an ongoing search for other healthcare options. An immigrant service provider recounted:

...people are against the public health...want it to be private...cannot trust any public services in their own country...they say 'because it is public it's not providing the service that you need.' So whatever the physician says, whatever the referral is, whatever the test ...they do not rely on that and they are always seeking another option (SIS3).

Many of the participants' comments related to chronic health conditions align with past research indicating some newcomers may not recognize the importance of preventive care to manage chronic conditions (Renfrew et al. 2013) and to maintain good health (Vargas Bustamante et al., 2010). Renfrew et al. (2013) observed that some immigrants did not understand diabetes as a chronic disease that cannot be cured and requires ongoing management. In addition, some newcomers prefer to rely on traditional healthcare approaches (Council of Agencies Serving South Asians, 2008). Lebanese healthcare providers pointed out

Border Crossing 
that Syrian refugees were relying on traditional remedies and not seeking medical care until the problem was advanced, resulting in poorer health outcomes (Dumit \& HoneinAbouHaidar, 2019). These diverse healthcare beliefs may result in delayed access to healthcare for the treatment of a minor problem until it requires urgent treatment. However, culture and beliefs can also contribute to maintaining social relations and encompass the capacity to frame conventional biomedical knowledge to support healthcare access in accordance with social values (Napier et al., 2014).

\section{Theme 9: Work, School, Childcare and Transportation Demands}

Although several service providers expressed concerns about healthcare access, barriers related to lack of childcare, transportation, and other household demands; newcomers themselves did not often mention these barriers. Many comments reflected the interconnectedness of these issues. An immigrant service provider explained, "We have to make a lot of tactic for the client to go...language barrier, transportation and daycare...if the husband is going to work...the mother cannot...go by bus, especially in this cold weather... they have 3 or 4 children...need childcare... and transportation" (SIS2).

Many newcomers sought conveniently located physicians to overcome transportation difficulties. An immigrant from the Middle East stated, "I am looking for one (physician) nearby our house because we don't have our own vehicle and we always need to get the bus to go so if it is near our house it is easy for us in winter" (SI1). Often, newcomer women were not as quick as their husbands to get their driver's license and thus relied on their husbands for transportation, as mentioned by an immigrant from the Middle East, "I am working so I have not much time to drive them... and their mother is not knowing how to drive...so I am everything for them, I am earning too and if there is something like going to the doctor I arrange their trips too" (SI2).

Many newcomers had very busy schedules to accomplish the tasks needed to successfully establish their new lives. This often made it difficult to attend healthcare appointments as recounted by an immigrant from the Middle East:

Right now I don't have time (for a preventative healthcare appointment) ... On the weekdays always in my work place I can't pick a phone and call anyone. I can if I get permission from my director...I always start from one thing to another, next week I am booked for my driving class lesson and theory, then next week one final exam (SI1).

Participant experiences reflect many of the same practical healthcare barriers identified in previous research, such as lack of transportation (Kilbride, 2010; Alwan et al., 2020) and childcare (Sethi, 2013). Newcomers may have difficulty arranging transportation or taking time off work to attend medical appointments (Council of Agencies Serving South Asians, 2008; O’Mahony \& Donnelly, 2007; Sanmartin \& Ross, 2006). Overall, newcomer families are coping with a multitude of daily tasks with limited social support and transportation options that make it a challenge to attend healthcare appointments.

Much of the comparative research mentioned above involved study participants from nonWestern countries and closely aligned with the experiences of ethnic minority newcomers from low- and middle-income countries in the current study. The current study also enrolled families from the US and Western Europe that did not report the same challenges as ethnic 
minority newcomers. They embodied biomedical healthcare perspectives and host language skills, and had privileged socioeconomic backgrounds, giving them greater capacity to effectively interact with the healthcare system. This suggests that ethnic minority newcomers to Saskatchewan are a vulnerable population at risk of not having sufficient access to healthcare to maintain optimal health due to social inequality. Although not intentional, the Saskatchewan healthcare system is contributing to the creation of a disadvantaged health status group by not responding to changing population needs. The results suggest consideration should be given to formal processes to ensure the timely provision of healthcare information, as well as responsive healthcare services, including convenient access and primary healthcare sites that offer comprehensive care in a culturally responsive manner with embedded interpretation services.

All the nine themes identified in the study permeate healthcare structures to negatively affect healthcare seeking behaviours. Although it is evident that issues extensively intertwine, each newcomer and their family will have their own unique experience due to personal backgrounds associated with country of origin that encompass gender, socio-cultural norms, and social-status, which can translate into opportunities, discrimination, stronger or weaker peer support networks, etc (Stepanikova \& Oates, 2017). These aspects can result in inequalities and health disparities related to ethnicity and age (Williams, 1999). Therefore, the assumption that newcomers encounter the same barriers and experience similar struggles, would be a narrow view of this complex problem. Our findings illustrate both user and provider perspectives on healthcare barriers that need to be recognised and further explored to understand, for example, how discriminatory attitudes impact perceived barriers to healthcare. Discrimination, stigma, and bias can directly affect healthcare seeking behaviours, as well as contribute to health disparities (White, Lawrence, Tchangalova et.al, 2020).

One of the study strengths is the inclusion of diverse newcomer groups, allowing for the exploration of a range of experiences across cultural, national, and socioeconomic factors that expose real concerns about the lack of culturally responsive care. Further, including service providers in the study allowed for the inclusion of both care providers' and receivers' perspectives. The study reveals gaps in Saskatchewan's healthcare system and suggests insights about healthcare access that can enhance understanding of cultural values, improve diagnostic accuracy and enhance patient engagement in biomedical treatments to improve newcomer health outcomes. The study's weakness lies in its limited urban scope, as newcomers in more rural and remote areas outside Regina and Saskatoon, may have experienced different or more intense challenges.

\section{Conclusion}

Newcomer families continue to experience numerous barriers to healthcare, including navigation difficulties, service awareness, language difficulties, low literacy, interpretation issues, limited appointment times, cultural and gender-specific needs, chronic disease perspectives, and busy schedules. Ethnic minority newcomers from low-to middle-income countries more commonly experienced challenges with accessing healthcare, as compared to newcomers from Western Europe and the US. Some immigrants were at a disadvantage compared to refugees due to not having a case manager to help them navigate and often experienced delayed access to provincial health benefits programs. These barriers resulted in treatment delays, inappropriate treatment, and in some cases unnecessary pain and suffering.

Border Crossing 
Immigration is changing the cultural dynamics of Saskatchewan. Strategic healthcare planning always mentions moving towards a patient-centered system, but has yet to move substantially beyond the Western Eurocentric viewpoint to truly transcultural planning for an increasingly diverse population. The incorporation of culturally responsive programming across multiple healthcare sites should include the availability of interpretation services, cultural competency staff training, and critical reflection of services that respond to diverse needs to ensure that the Saskatchewan healthcare system does not perpetuate or create health disparities.

Consideration should be given to ethnicity, embodied and attributed social status and role, gender, etc. when designing and introducing policies and healthcare interventions.

\section{References}

Access Alliance. (2011). The Global City: Newcomer Health in Toronto 2011. Toronto, ON: Toronto Public Health. Retrieved from: http://www.toronto.ca/health/map/newcomer.htm

Alwan, R., Schumacher, D., Cicek-Okay, S., Jernigan, S., Beydoun, A., Salem, T., Vaughn, L., \& Gopichandran, V. (2020). Beliefs, perceptions, and behaviors impacting healthcare utilization of Syrian refugee children. PLoS ONE, 15(8), e0237081. https://link.gale.com/apps/doc/A631879505/EAIM?u $=$ usaskmain\&sid $=$ EAIM\&xid $=4 \mathrm{db} 54300$

Council of Agencies Serving South Asians (CASSA). (2008). Summary report: South Asian and health- A roundtable to identify gaps in health promotion for South Asian communities in Ontario. Toronto, ON: CASSA. Retrieved from: http://www.cassaonline.com/index3/downloads/Projects/South_Asians_ Health_Summary_Report.pdf.

Cruz, E., \& Higginbottom, G. (2013). The use of focused ethnography in nursing research. Nurse Researcher, 20(4), 36-43.

Cypress, B. (2017). Rigor or Reliability and Validity in Qualitative Research: Perspectives, Strategies, Reconceptualization, and Recommendations. Dimensions of Critical Care Nursing, 36(4), 253-263.

DuBard, C., \& Gizlice, Z. (2008). Language spoken and differences in health status, access to care, and receipt of preventive services among US Hispanics. American Journal of Public Health, 98(11), 2021 2028.

Dumit, N., \& Honein-AbouHaidar. (2019). The Impact of the Syrian Refugee Crisis on Nurses and the Healthcare System in Lebanon: A Qualitative Exploratory Study. Journal of Nursing Scholarship, 51, 3, 289-298.

Goggins, K. (2008). More than words: Healthcare interpreters play key role in quality care. Cross Currents, 11(3), 14-15. Retrieved from: http://www.camh.net/Publications/Cross_Currents/Spring_2008/ morethanwords_crcu spring $08 . h t m l$

Guruge, S., Sidani, S., Illesinghe, V. et al. (2018). Healthcare needs and health service utilization by Syrian refugee women in Toronto. Conflict and Health, 12, 46. https://doi.org/10.1186/s13031-018-0181-x

Green, J., \& Thorogood, N. (2007). Qualitative Methods for Health Research. Thousand Oaks, California: Sage Publications.

Groleau, D., \& Kirmayer, L. (2004). Sociosomatic theory in Vietnamese immigrants' narratives of distress. Anthropology and Medicine, 11,117-133.

Hansson, E., Tuck, A., Lurie, S., \& McKenzie, K., for Services Systems Advisory Committee Task Group, Mental Health Commission of Canada. (2009). Improving mental health services for immigrant, refugee, ethno-cultural and racialized groups: Issues and options for service improvement. Toronto, ON: Centre for Addiction and Mental Health. Retrieved from: http://www.mentalhealthcommission.ca/Site CollectionDocuments/News/en/IO.pdf

Hengstermann, M. (2016). A Comprehensive Interaction between Illness and Culture: Recognition and Treatment of Evil Eye in rural San Marcos, Guatemala. Freie Universität, Berlin.

Higginbottom, G. (2013). Guidance on performing focused ethnographies with an emphasis on healthcare research. The Qualitative Report, 18, 1-16. Retrieved from: http://www.nova.edu/ssss/QR/QR18/ higginbottom17.pdf.

Juckett, G., \& Unger, K. (2014). Appropriate use of medical interpreters. American Family Physician, 90(7), 476-480. 
170 Newcomer challenges with accessing healthcare services in Saskatchewan, Canada

Karago-Odongo, J. (2008). Immigrant Women Living with HIV/Aids: Their Barriers as Experienced by Service Providers. (Doctoral Dissertation). Retrieved from: http://digitalcommons.ryerson.ca/ dissertations $/ 91$.

Kilbride, K.M. (2010). Speaking with immigrant seniors and their families - Summary. Toronto, ON: Centre for Excellence in Research on Immigration and Settlement (CERIS). Retrieved from: http://www.isgw.ca/storage/project-wisdom/Kilbride $\% 20-\% 20$ Phase $\% 201 \% 20$ full $\% 20$ report.pdf

Knoblauch, H. (2005). Focused ethnography. Forum: Qualitative Social Research, 6(3), Art. 44. Retrieved from: http://nbn-resolving.de/urn:nbn:de:0114-fqs0503440.

Lane, G., Farag, M., White, J., Nisbet, C., \&Vatanparast, H. (2018). Chronic Health Disparities among Refugee and Immigrant Children in Canada. Applied Physiology, Nutrition, and Metabolism,

Mayan, M. (2009). Essentials of qualitative inquiry. Walnut Creek, CA: Left Coast Press.

Morse, J., \& Field, P. (1995). Qualitative Research Methods for Health Professionals (2nd ed.). Thousand Oaks, CA: Sage.

Morse, J. (2015). Critical Analysis of Strategies for Determining Rigor in Qualitative Inquiry. Qualitative Health Research, 25(9) 1212-1222.

Munger, F., Popal, Z., Jayaratnam, J., \& Rutherford, E. (2010). Focus groups report (Appendix 3). The Culture, Diversity and Equity Project: Ensuring accessibility to appropriate healthcare for all. Toronto, ON: Central East Local Health Integration Network. Retrieved from:http://www.centraleastlhin.on.ca/ uploadedFiles/Home_Page/Board_of_Directors/Board_Meeting_Submenu/CDE_Appendix_3_Foc us_Groups.pdf.

Napier, D.; Ancarno; C., Butler, B., et al. (2014). Culture and Health. The Lancet, 384: 1607-1639.

Neufeld, A., Harrison, M., Stewart, M., Hughes, K., \& Spitzer, D. (2002). Immigrant women: making connections to community resources for support in family caregiving. Qualitative Health Research, 12, 751-768.

O’Mahony, J., \& Donnelly, T. (2007). Health care providers' perspective on the gender influences on immigrant women's mental health care experiences. Issues in Mental Health Nursing, 28, 1171-1188.

O’Mahony, J., \& Donnelly, T. (2013). How does gender influence immigrant and refugee women's postpartum depression help-seeking experiences? Journal of Psychiatric and Mental Health Nursing, 20, 714-725.

Renfrew, M., Taing, E., Cohen, M., Betancourt, J., Pasinski, R., \& Green, A. (2013). Barriers to care for Cambodian patients with diabetes: Results from a qualitative study. Journal of Health Care for the Poor and Underserved, 24(2), 633-655.

Roper, J., \& Shapira, J. (2000). Ethnography in nursing research. Thousand Oaks, CA: Sage.

Sanmartin, C., \& Ross, N. (2006). Experiencing difficulties accessing first-contact health services in Canada. Healthcare Policy, 1(2), 103-119. Retrieved from: https://www.ncbi.nlm.nih.gov/pmc/articles/PMC25 $85333 /$

Sethi, B. (2013). Service delivery on rusty health care wheels: Implications for visible minority women. Journal of Evidence-Based Social Work, 10(5), 522-532.

Smith, V. (2001). Ethnographies of work and the work of ethnographers. In P. Atkinson, A. Coffey, S. Delamont, J. Lofland \& L. Lofland (Eds.), Handbook of ethnography (pp.220-233). Thousand Oaks, CA: Sage.

Son, J. (2013). Assimilation and health service utilization of Korean immigrant women. Qualitative Health Research, 23(11), 1528-1540.

Stepanikova, I., Oates, G. (2017). Perceived discrimination and privilege in health care: The role of socioeconomic status and race. Am J Prev Med., 52(1S1), S86-S94.

doi: 10.1016/j.amepre.2016.09.024. PMID: 27989297; PMCID: PMC5172593.

Szajna, A., \& Ward, J. (2015). Access to health care by refugees: a dimensional analysis. Nursing Forum, 50, $83-89$.

Vargas Bustamante, A., Chen, J., Rodriguez, H., Rizzo, J., \& Ortega, A. (2010). Use of preventive care services among Latino subgroups. American Journal of Preventive Medicine, 38(6), 610-619.

Weerasinghe, S., \& Mitchell, T. (2007). Connection between the meaning of health and interaction with health professionals: caring for immigrant women. Health Care for Women International, 28, 309-328.

Williams, D. (1999). Race, socioeconomic status, and health. The added effects of racism and discrimination. Ann N Y Acad Sci., 896, 173-88. doi: 10.1111/j.1749-6632.1999.tb08114.x. PMID: 10681897. 
White, K., Lawrence, J., Tchangalova, N., Huang, S., Cummings, J. (2020). Socially-assigned race and health: a scoping review with global implications for population health equity. Int J Equity Health., 19(1), 25. doi: 10.1186/s12939-020-1137-5. PMID: 32041629; PMCID: PMC7011480.a

Woodgate, R., Shiyokha Busolo, D., Crockett, M., Dean, R., Amaladas, M., \& Plourde, P. (2017). A qualitative study on African immigrant and refugee families' experiences of accessing primary health care services in Manitoba, Canada: it's not easy! International Journal for Equity in Health, 16, 5.

Wu, Z., Penning, M., \& Schimmele, C. (2005). Immigrant status and unmet health care needs. Canadian Journal of Public Health, 96(5), 369-373.

Young, D., Spitzer, D., \& Pang, F. (1999). Understanding the health care needs of Canadian immigrants. Report submitted to the Prairie Centre for Excellence on Immigration and Integration. Retrieved from: http://pcerii.metropolis.net/virtual\%20library/finalreports/young99.htm

Yun, K., Fuentes-Afflick, E., Curry, L., Krumholz, H, \& Desai, M. (2013). Parental immigration status is associated with children's health care utilization: Findings from the 2003 new immigrant survey of US legal permanent residents. Maternal and Child Health Journal, 17, 1913-1921.

\section{Appendix A}

\section{Parent Interview Guide}

How did you find out about accessing health care services in Canada?

Please describe the first time you took your children to a health care appointment after arriving in Canada. Did your child have a health problem? What did the physician ask about? What types of tests or measurements were done?

After that first visit to the physician, have you taken your children to see a physician again? Can you tell me about it? (Probe for location, health problem, treatment offered, follow through with treatment) Did the treatment work well -why? Was the treatment difficult to follow or work poorly? What treatments did you expect to receive for the health problem that your child did not receive? Have you sought out any other therapy or treatment? Would you like your child to receive any other therapy or treatments?

Do you take your children to the public health clinic to access any services? Please describe. (Probe for immunizations, healthy child check up)

Has one of your children been hospitalized in Canada? Can you tell me about it? How did you manage to care for child when s/he came home? Were you offered any additional health services to help your child at home?

Does your family qualify for any special health programs, such as Supplementary Health Program, Family Health Benefits or Special Support for Drug Coverage? Please describe. Which benefits do you use? (Probe for children's dental care, eye care, prescription drug coverage, medical supplies, foot care, hearing services, chiropractic treatments)

Do you ever find it difficult to access health care services for your children? Please describe.

Are some health care services easier to access than others? Please describe.

What kind of changes do you think could be made to the Canadian health care system so it could provide better service to your family and other newcomers? 


\section{Service Provider Guide}

What barriers do you believe exist for immigrants and refugees attempting to access health care in Canada?

What strategies for dealing with these barriers have you observed among immigrants/refugees? Do you believe these strategies were culturally-based?

What changes could be made to reduce or eliminate these barriers?

Is there anything else you can think of that could be changed to make the health care system more accessible to immigrants and refugees?

Are there any other things that could be done or changed that could contribute to the health of immigrants, particularly children (e.g. alternative programs/ services, changes in policy etc.)?

Do you feel that the recent changes to interim federal health care service coverage has had an impact on refugees? If so, can you describe the impact? (The interim federal health care service changes have restricted access to health services by some groups of refugees, including those from designated countries of origin and privately sponsored refugees. Interim federal health care services are described below.)

Are you aware of any programs or services that are aimed specifically at making health care more accessible to immigrants or connecting immigrants to health resources?

Do you make referrals to those programs or services? If so, do you think the individuals followed-up on them? Why or why not? If not, what makes if difficult? What could make it easier or more straightforward?

What role (if any) do you think traditional, ethnic or alternative healers or healing practices can play in the health of immigrants?

Do you feel there are opportunities for immigrants to provide input into health care service delivery? If so, what are they? Do you feel these opportunities are adequate? If not, what could be changed to create such opportunities?

What do you feel your organization can do to impact the health of immigrants and their children? To improve access to health care? 